\title{
EDITORIAL
}

\section{From mechanisms to management}

\author{
In examining disease, we gain wisdom about anatomy \\ and physiology and biology. In examining the person \\ with disease, we gain wisdom about life.
}

the Primer serves as a signpost of where the field currently is and where it's heading
Much progress has been made in medical research in the 30 years since neurologist Oliver Sacks penned these words in The Man Who Mistook His Wife for a Hat and Other Clinical Tales. This wisdom - which encompasses more than anatomy, physiology and biology has advanced at a rate at which even the most dedicated student would struggle to keep pace. Medical research is a multidisciplinary endeavour, producing a continuous stream of new techniques, data and analyses. On top of this, it can be difficult to decipher the discipline-specific language and concepts used by the different researchers studying related problems, from biochemists, computer modellers and bioengineers to clinicians.

At Nature Reviews Disease Primers, we commission leading international experts from across the research trajectory - including laboratory scientists and clinicians - to summarize our current understanding of a particular disease or disorder with a global perspective. Each Primer article includes the same major sections: epidemiology, mechanisms, pathophysiology, diagnosis, screening, prevention, management and patient quality of life. Indeed, all Primers have a common goal: they not only describe the current state of the art, but are also forward-looking. In the final 'Outlook' section of each article, the authors describe the key research questions to be answered and the major challenges that lie ahead in the next decade. For newcomers to the field, the Primer paints the landscape and logically explains all of the major concepts in a particular disease or disorder. For established researchers, the
Primer serves as a signpost of where the field currently is and where it's heading.

Alongside each of these articles you will find an accompanying PrimeView - a poster that gives an illustrated summary of the Primer. Together, we hope that the Primer and PrimeView will provide readily accessible introductions to each topic for readers from all disciplines.

The Oxford English Dictionary defines disease as "a condition of the body, or of some part or organ of the body, in which its functions are disturbed or deranged". As should be clear from our first articles, Nature Reviews Disease Primers will cover 'classic' diseases such as melanoma, systemic sclerosis and Huntington disease; however, we'll also address other important healthrelated topics, including the consequences of ageing such as menopause, which increases the risk for cardiovascular disease and osteoporosis. Our scope also includes behavioural conditions, developmental disorders and nutritional deficiencies - all of which have important consequences for human health - as well as rare, communicable and non-communicable diseases.

In his work, Sacks undoubtedly shared wisdom about anatomy, physiology and biology and, through his candour, wisdom about his life and experiences; he always emphasized that the patient should be at the heart of medical research. Earlier this year, he announced in The New York Times that he has metastatic ocular melanoma with a poor prognosis. Although medical research will not outpace his condition, future generations will benefit from the scientific discoveries being made in melanoma, and many other diseases, every day. Nature Reviews Disease Primers will summarize the burgeoning scientific wisdom about disease, which, in time, will translate into improvements in patients' lives.

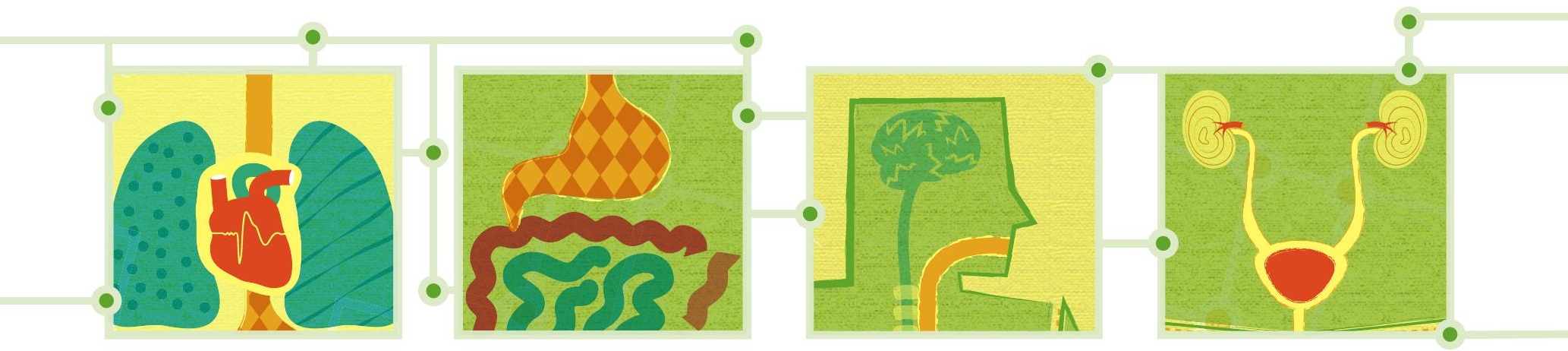

